\title{
Post-Training Intra-Basolateral Amygdala Infusions of Norepinephrine Enhance Consolidation of Memory for Contextual Fear Conditioning
}

\author{
Ryan T. LaLumiere, Thea-Vanessa Buen, and James L. McGaugh \\ Center for the Neurobiology of Learning and Memory and Department of Neurobiology and Behavior, University of California, Irvine, Irvine, California \\ 92697-3800
}

\begin{abstract}
Post-training infusions of drugs, including noradrenergic agonists and antagonists, into the basolateral amygdala (BLA) influence the consolidation of memory for training in several tasks, including inhibitory avoidance. There is, however, conflicting evidence concerning whether post-training intra-BLA drug infusions modulate the consolidation of contextual fear conditioning (CFC). In the present study, norepinephrine (NE) was infused bilaterally into the BLA of male Sprague Dawley rats immediately after training on two CFC tasks: a Y-maze and a straight alley. Post-training intra-BLA infusions enhanced memory of CFC training in the Y-maze, as assessed by percentage of time spent freezing and shock arm entrance latencies. Post-training intra-BLA infusions of NE enhanced $48 \mathrm{hr}$ retention of CFC training in the straight alley, as assessed by shock compartment entrance latencies and the number of shocks required to learn to avoid entering the shock compartment. These findings indicate that the consolidation of memory for CFC, like that for inhibitory avoidance training, is influenced by post-training neuromodulatory influences within the BLA. Thus, the findings provide additional evidence consistent with the hypothesis that the BLA has a general role in modulating memory consolidation.
\end{abstract}

Key words: amygdala; memory consolidation; norepinephrine; contextual fear conditioning; inhibitory avoidance; learning

\section{Introduction}

Extensive evidence indicates that noradrenergic activation within the basolateral amygdala (BLA) plays a critical role in modulating memory consolidation. Infusions of $\beta$-adrenoceptor agonists, including norepinephrine (NE), into the BLA after training on inhibitory avoidance (IA) or water maze spatial tasks enhance rats' long-term memory of the training (Ferry and McGaugh, 1999; Ferry et al., 1999a; Hatfield and McGaugh, 1999; Power et al., 2002). Studies using IA training have demonstrated that noradrenergic influences in the BLA mediate the memorymodulating effects of drugs affecting opioid peptidergic, GABAergic, and glucocorticoid systems (Introini-Collison et al., 1989, 1994; Quirarte et al., 1997). Drugs that enhance memory when infused into the BLA after training, including the opiate antagonist naloxone and the GABAergic antagonist bicuculline, enhance NE release in the amygdala, and drugs that impair consolidation, including the GABAergic agonist muscimol, decrease amygdala NE release (Quirarte et al., 1998; Hatfield et al., 1999). Moreover, the amount of amygdala NE released after IA training correlates highly with subsequent retention performance (McIntyre et al., 2002).

\footnotetext{
Received March 3, 2003; revised June 3, 2003; accepted June 4, 2003.

This work was supported by National Institutes of Health and United States Public Health Service Grant MH15256 to J.L.M. We thank Emmanuel Pizano, Nicole Batard, Arthur Han, Linda Nguyen, Tudy Atia, Naomi Raymundo, Stacy Yu, Lindsay Yourman, and Mallika Thomas for their excellent technical assistance.

Correspondence should be addressed to Ryan T. LaLumiere, Center for the Neurobiology of Learning and Memory, University of California, Irvine, Irvine, CA 92697-3800. E-mail: rlalumie@uci.edu.

Copyright $\odot 2003$ Society for Neuroscience $\quad$ 0270-6474/03/236754-05\$15.00/0
}

Substantial evidence also suggests that the BLA is involved in the acquisition and retention of Pavlovian contextual fear conditioning (CFC) (Helmstetter and Bellgowan, 1994; Maren et al., 1996; Muller et al., 1997; Vazdarjanova and McGaugh, 1998). Because both IA and CFC training involve learning that contextual cues are associated with footshock, and because of evidence that retention of both types of training involve the BLA, posttraining infusions of drugs into the BLA might be expected to have comparable effects on memory consolidation for both training types. The evidence concerning this issue is conflicting. Wilensky et al. (2000) reported that in confirmation of previous findings (Brioni et al., 1989; Izquierdo et al., 1992), post-training intra-BLA infusions of the GABAergic agonist muscimol impaired IA retention. However, these investigators also reported that post-training intra-BLA infusions of muscimol did not impair retention of CFC (or Pavlovian tone fear conditioning). They interpreted those findings as suggesting that IA and CFC differ in their neurobiological bases and that the BLA has different roles in enabling memory for these two kinds of fear-based training (Wilensky et al., 2000). However, several studies have reported that post-training intra-BLA drug infusions can impair or enhance retention of CFC. Infusions of cholinergic or histaminergic agonists enhance CFC retention, and infusions of histaminergic antagonists, lidocaine, or tetrodotoxin (TTX) impair CFC retention (Sacchetti et al., 1999; Vazdarjanova and McGaugh, 1999; Passani et al., 2001; Cangioli et al., 2002).

To investigate this issue further, the present experiments examined the effects of post-training intra-BLA infusions of NE on 
retention of CFC using two types of training apparatuses and several indices of retention. NE was used to investigate influences on memory consolidation in view of the substantial evidence that noradrenergic activation in the BLA plays a critical role in influencing consolidation of memory for IA training (Ferry et al., 1999a,b) and that GABAergic effects on memory consolidation are mediated by influences on NE release in the amygdala (Hatfield et al., 1999).

\section{Materials and Methods \\ Subjects}

Male Sprague Dawley rats ( $\sim 300$ gm body weight at the time of surgery; Charles River Laboratories, Wilmington, MA) $(n=134)$ were used. They were housed individually, maintained in a temperature-controlled environment $\left(22^{\circ} \mathrm{C}\right)$ on a $12 \mathrm{hr}$ light/dark cycle (lights on at 7:00 A.M.) with food and water ad libitum, and given $7-8 \mathrm{~d}$ to acclimatize to the vivarium before undergoing surgery. Behavioral procedures began $6-9 \mathrm{~d}$ after surgeries. All methods used were in compliance with National Institutes of Health guidelines for care of laboratory animals and were approved by the University of California, Irvine, Institutional Animal Care and Use Committee.

\section{Surgery}

The rats were anesthetized with sodium pentobarbital (50 mg/kg, i.p.) and given atropine sulfate $(0.1 \mathrm{mg}$, i.p.) to prevent respiratory congestion as well as saline $(3.0 \mathrm{ml}$, s.c.) to prevent dehydration during surgery. Supplemental doses of sodium pentobarbital were given as needed during surgery, although the surgeries were usually short and the animals seldom required supplements. The rats were then placed in a small animal stereotaxic instrument (Kopf Instruments, Tujunga, CA). Two surgical screws were implanted into the skull as anchors, and guide cannulas aimed at the BLA were implanted bilaterally, $2.8 \mathrm{~mm}$ posterior and 5.0 $\mathrm{mm}$ lateral to bregma and $6.5 \mathrm{~mm}$ ventral to the skull surface (Paxinos and Watson, 1997). The nose bar was maintained at $-3.5 \mathrm{~mm}$ relative to the interaural line. The guide cannulas were constructed of 23 gauge stainless steel tubing cut to a length of $15.0( \pm 0.02) \mathrm{mm}$. The cannulas and screws were affixed to the skull with dental cement. Insect pins (15 $\mathrm{mm}$ long 00 insect dissection pins) were inserted into the cannulas to maintain patency and were removed only for the infusions. After the surgery, the rats were placed in an incubation chamber where they were kept warm until they awoke. The animals were then returned to their home cages and checked on the days after surgery to ensure that their wounds were not infected and to replace any missing cannula pins.

\section{Behavioral procedures}

Experiment 1. The behavioral apparatus used in the first experiment was a Y-maze consisting of three trough-shaped alleys with stainless steel floors and walls. The alleys were separated by $120^{\circ}$ and joined in the middle. Each alley was $50 \mathrm{~cm}$ long and $18 \mathrm{~cm}$ deep and was covered by a clear Plexiglas lid. One alley, designated the shock alley, had vertical black stripes along the sides. The two nonshock alleys had horizontal white stripes along the sides. The plates of the shock alley were connected to an AC shock generator (Lafayette Instruments, Lafayette, IN) controlled by a timer. A video camera mounted $1 \mathrm{~m}$ above the maze recorded the rats' behavior.

Each rat was handled for $1 \mathrm{~min}$ per day for $3 \mathrm{~d}$ before the start of training. All training and testing occurred between 11:00 A.M. and 5:00 P.M. Immediately after the training and testing of each animal, the apparatus was cleaned with a $10 \%$ ethanol solution. On day 1 , the habituation day, each rat was placed at the end of one of the nonshock alleys and permitted to explore the entire maze freely for $8 \mathrm{~min}$. On day 2, the training day, the shock alley was blocked off from the rest of the maze by a door. The rat was placed directly in the shock alley and retained there for a total of $1 \mathrm{~min}$. The rat received two footshocks ( $1 \mathrm{sec}, 0.5 \mathrm{~mA}, \mathrm{AC}$ ), the first after a delay of $15 \mathrm{sec}$ and the second after another delay of $15 \mathrm{sec}$. Another group of animals (no-shock controls) was placed in the shock alley and retained there for a total of $1 \mathrm{~min}$ and given no footshocks. For all groups, the rat was then removed and given the appropriate drug infusion, either immediately (shock and no-shock animals) or $3 \mathrm{hr}$ later (shock animals). On day 3 , the testing day, each rat was placed at the end of one of the nonshock alleys and permitted to explore the entire maze freely for $8 \mathrm{~min}$. The alley in which the rats were placed was counterbalanced according to the habituation day arm placement. Footshock was not administered during the test. The time spent freezing and latency to enter the shock alley were used as indices of retention of the footshock training. Freezing was defined as the cessation of all movement except what was necessary for respiration. A scorer, blind to the drug conditions of the animals, viewed the videotapes of the animals' tests and measured their freezing using a stopwatch.

Experiment 2. The apparatus used in the second experiment was an IA box. However, the apparatus was used for fear conditioning rather than conventional IA training. The box was a $91 \mathrm{~cm}$ long, $20 \mathrm{~cm}$ deep, troughshaped alley with stainless steel covering the floor and walls. The box was divided into two compartments: a safe compartment $(31 \mathrm{~cm}$ long) illuminated by a tensor lamp and a darkened shock compartment $(60 \mathrm{~cm}$ long). The two compartments differed in their textures; the safe compartment was constructed of clear plastic, and the shock compartment was constructed of stainless steel. The compartments were separated by a door that retracted into the floor.

Each rat was handled 1 min per day for $3 \mathrm{~d}$ before the start of training. All training and testing occurred between 10:00 A.M. and 4:00 P.M. On day 1 , the training day, each rat was placed directly into the shock compartment, with the retractable door blocking the entrance to the safe compartment, and retained there for a total of $1 \mathrm{~min}$. The rat received two footshocks ( $1 \mathrm{sec}, 0.5 \mathrm{~mA}, \mathrm{AC}$ ), the first after a delay of $15 \mathrm{sec}$ and the second after another delay of $15 \mathrm{sec}$. Therefore, the training procedures in this experiment and the experiment described previously were identical. Immediately after the training, each animal was removed from the apparatus and given its appropriate intra-BLA infusions.

Retention was tested $48 \mathrm{hr}$ later. Each animal was placed into the safe compartment and permitted to explore the box freely. The animal's initial latency to step into the shock compartment with all four paws was measured. After the animal stepped through, continuous multiple-trial inhibitory avoidance (CMIA) training was used to assess "savings" of the original training. A continuous footshock $(0.3 \mathrm{~mA}$, AC) was administered in the shock compartment and remained on until the rat returned to the safe compartment. The rat received a footshock on each complete reentry of the shock compartment, and the training was terminated when the rat remained in the safe compartment for the criterion time of 200 sec. The number of shocks required for reaching the criterion was recorded and used as a measure of memory for the original training. If the animal's initial latency was $200 \mathrm{sec}$ or higher, a score of zero was given for the CMIA training.

\section{Drugs and infusion procedures}

Norepinephrine was obtained from Sigma (St. Louis, MO). NE was dissolved in a phosphate-buffer solution (0.1 M PBS, pH 7.4). Two doses of NE were used, 0.3 and $1.0 \mu \mathrm{g}$, except in the $3 \mathrm{hr}$ post-training group and the no-shock group, in which only the effective dose $(1.0 \mu \mathrm{g})$ was used.

To infuse the drug or vehicle into the BLA, PE-20 polyethylene tubing was connected to a $10 \mu \mathrm{l}$ Hamilton syringe, and a 30 gauge dental needle was cemented to the other end of the tubing. This infusion needle was then bent to a length of $17 \mathrm{~mm}$ to extend $2 \mathrm{~mm}$ beyond the end of the guide cannula into the BLA. The tubing was first filled with distilled water. A small air bubble was then pulled in and the drug or vehicle was then pulled in. The Hamilton syringe was driven by an automated syringe pump (Sage Instruments, Boston, MA) at the rate of $0.38 \mu \mathrm{l} / \mathrm{min}$. To perform the infusion, the animal's pins were removed from the cannulas, and the infusion needles were inserted. The syringe pump was turned on for $32 \mathrm{sec}$ to give an infusion volume of $0.2 \mu \mathrm{l}$ on each side. The needles were then left in place for an additional $35 \mathrm{sec}$ to permit the liquid to diffuse. After the infusions, the needles were removed and the animals were returned to their home cages.

\section{Histology}

The animals were killed with an overdose of sodium pentobarbital (100 $\mathrm{mg} / \mathrm{kg})$ and perfused through the heart with physiological saline $(0.9 \%$ $\mathrm{NaCl}$ ) and then formaldehyde (4\%). The brains were removed and 

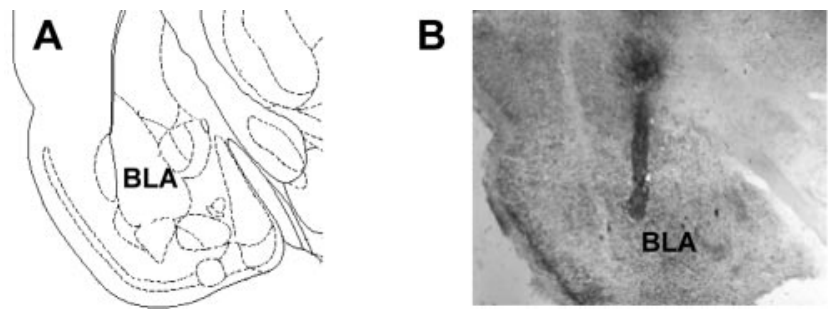

Figure 1. A, Diagram of rat basolateral amygdala and adjacent structures (Paxinos and Watson, 1997). B, Representative photomicrograph of needle track terminating in the BLA. Only data from animals that had needle tracks terminating in the BLA and had no lesions in the surrounding BLA tissue were included in the analyses.

stored in formaldehyde for a minimum of $24 \mathrm{hr}$. At least $48 \mathrm{hr}$ before the brains were sectioned, the brains were transferred to a $25 \%$ sucrose solution. The brains were sectioned $(40 \mu \mathrm{m})$ on a freezing microtome and mounted onto gelatin-subbed slides. The sections were then stained with thionin. The location of the infusion needles was determined by examining the sections under a microscope and using a rat brain atlas (Paxinos and Watson, 1997). Only animals that had needle tips located within the BLA and had no lesions around the needle tips were used in the final analysis.

\section{Statistics}

The measures used in experiment 1 were analyzed with a one-way ANOVA (immediately after training infusions) or a $t$ test ( $3 \mathrm{hr}$ after training infusions only) with a dose of NE administered as the betweensubjects variable. Fisher's post hoc tests were performed to determine the source of detected significances in the ANOVA. $p$ values of $<0.05$ were considered significant. The two measures used in experiment 2 were analyzed with $t$ tests. $p$ values of $<0.05$ were considered significant. All measures are expressed as mean \pm SEM. The number of animals in each group is indicated in the figure legends.

\section{Results \\ Histology}

Figure 1 shows a representative photomicrograph of a cannula tip dorsal to the BLA and a needle track terminating in the BLA. In experiment 1, 62 of 89 animals had correct bilateral infusion needle placements. In experiment 2, 31 of 45 animals had correct bilateral infusion needle placements.

\section{Experiment 1}

The results of animals given immediate post-training intra-BLA infusions are shown in Figure 2. Figure $2 A$ shows the percentage of time spent freezing on the retention test day. A one-way ANOVA revealed a significant effect $\left(F_{(4,40)}=5.798 ; p<0.001\right)$. The percentage of time spent freezing was significantly higher for rats given the $1.0 \mu \mathrm{g}$ dose of $\mathrm{NE}$ compared with vehicle control animals $(p<0.005)$. Although the time spent freezing was measured in all three arms, it should be noted that the animals that froze did so exclusively in the start arm immediately after being placed there. Also, although percent freezing observed in the group given the higher NE dose was significantly greater than that of the vehicle controls, that NE group displayed freezing for only $\sim 15 \%$ of the time. The amount of time spent freezing during the test was not compared with the time spent freezing during the habituation because none of the animals froze during habituation. Figure $2 B$ shows the shock arm entrance latencies during the test. A one-way ANOVA revealed a significant effect $\left(F_{(4,40)}=\right.$ 7.699; $p<0.001)$. The latencies of the rats given the $1.0 \mu \mathrm{g}$ dose of NE were significantly longer than those of the vehicle control group and longer than those of both sets of no-shock controls (i.e., the vehicle and $1.0 \mu \mathrm{g}$ NE no-shock groups) ( $p<0.005$ for all three comparisons).

To assess the contribution of freezing to the shock compartment entrance latencies, the total amount of time spent freezing during the retention test was subtracted from the shock arm latencies during the test (Fig. 2C). A one-way ANOVA of this latency-without-freezing measure revealed a significant effect $\left(F_{(4,40)}=7.326 ; p<0.001\right)$. Thus, even with the time spent freezing removed from their entrance latencies, rats that received the $1.0 \mu \mathrm{g}$ dose of NE had significantly higher latencies compared with those of the vehicle controls as well as those of both noshock controls ( $p<0.005$ for all three comparisons). To determine whether the different latencies reflected differences between the groups that existed before training, the percentage change in shock arm entrance latencies from the habituation session to the retention test was calculated. A one-way ANOVA of this percentage change revealed a significant effect $(F=5.677 ; p<0.005$; data not shown). Rats that received the $1.0 \mu \mathrm{g}$ dose of NE had a significantly higher increase in latencies compared with vehicle controls and both no-shock controls $(p<0.005$ for all three comparisons).

Animals that received $3 \mathrm{hr}$ post-training infusions of either the vehicle or the $1.0 \mu \mathrm{g}$ infusion of NE did not differ in shock arm entrance latencies $\left(t_{(15)}=0.074 ; p>0.05\right.$; data not shown). The amount of time spent freezing was not measured in this experiment.

\section{Experiment 2}

Figure $3 A$ shows the animals' latencies to enter the dark shock compartment on the test day. The retention latencies of animals given the $1.0 \mu \mathrm{g}$ dose of NE were significantly longer than those of the vehicle controls $\left(t_{(29)}=2.261 ; p<0.05\right)$. Figure $3 B$ shows the number of shocks required for animals to reach the criterion of $200 \mathrm{sec}$ in the light compartment. Animals that received the 1.0 $\mu \mathrm{g}$ dose of NE required significantly fewer shocks to reach the criterion $\left(t_{(29)}=2.054 ; p<0.05\right)$.

\section{Discussion}

The findings of these experiments indicate that post-training infusions of NE administered into the BLA enhanced rats' retention of contextual fear conditioning, as assessed in two types of training tasks and several indices of retention. The finding that infusions administered $3 \mathrm{hr}$ after training did not affect retention indicates that the memory-enhancing effects of NE infused immediately after training are attributable to influences on memory consolidation. Because the post-training NE infusions did not affect the retention of the no-shock group, the enhanced retention of the rats receiving NE infusions and a shock was not the result of an influence of the NE infusions alone on the memory for shock arm confinement or on the subsequent retention performance.

These findings are consistent with extensive evidence that post-training intra-BLA infusions of NE, as well as drugs affecting the release of NE within the amygdala, enhance the consolidation of memory for IA training, as well as several other types of training (Quirarte et al., 1998; Ferry and McGaugh, 1999; Ferry et al., 1999a; Hatfield and McGaugh, 1999; Hatfield et al., 1999; Power et al., 2002). The findings provide additional evidence that the consolidation of CFC, like that of IA training, is modulated by post-training manipulation of BLA functioning, and that the BLA has the same role in the consolidation of memory for these two types of aversive training experiences (Vazdarjanova and McGaugh, 1999). The findings do not support the view that CFC and 


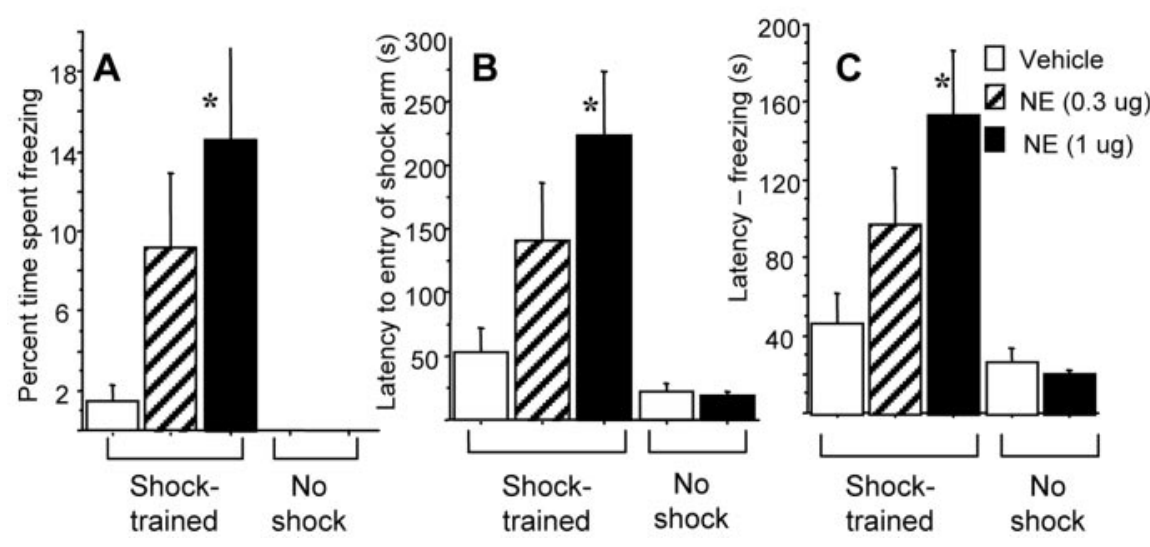

Figure 2. Enhanced retention of rats that received immediate post-training NE infusions into the BLA for Y-maze CFC. Groups are as follows (from left to right): vehicle shock (white bars; $n=10$ ), $0.3 \mu \mathrm{g} \mathrm{NE} \mathrm{shock} \mathrm{(hatched} \mathrm{bars;} n=8$ ), $1.0 \mu \mathrm{g} \mathrm{NE} \mathrm{shock}$ (black bars; $n=10$ ), vehicle no shock (white bars; $n=7$ ), and $1.0 \mu \mathrm{g} \mathrm{NE} \mathrm{no} \mathrm{shock} \mathrm{(black} \mathrm{bars;} n=10$ ). $A$, Mean percentage of time ( \pm SEM) spent freezing during the 8 min retention test on day 3. $B$, Mean latencies ( \pm SEM), in seconds, to first entry into the shock arm during retention test. C, Mean latencies without freezing ( \pm SEM), in seconds, during the 8 min retention test on day 3 . ${ }^{*} p<0.005$ compared with shocked vehicle controls and both no-shock controls. ing are consistent with those of studies using IA training and testing procedures in indicating that the latency to enter a compartment where footshock had been received provides clear evidence of the memory-enhancing effects of posttraining intra-BLA drug infusions.

Freezing behavior on the retention test is clearly not required for evidence of rats' retention of CFC training or for the enhancing effects of post-training intra-BLA infusions of NE on retention. However, in experiment 1 , the rats given post-training intra-BLA infusions of NE $(1.0 \mu \mathrm{g})$ also displayed significantly more freezing on the retention test, compared with that of the controls. Thus, the present findings, as well as those of other experiments using freezing as the index of retention of CFC, indicate that post-training intra-BLA infusions of drugs can enhance CFC retention as measured by freezing. Post-training
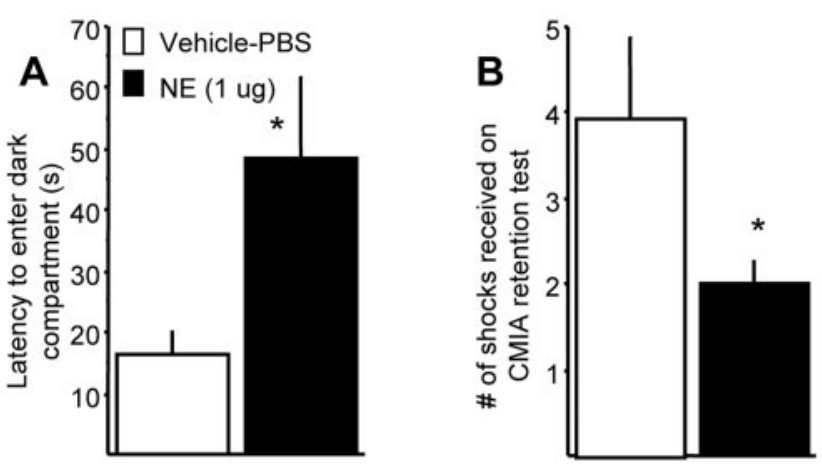

Figure 3. Enhanced retention of rats that received immediate post-training NE infusions into the BLA for straight-alley CFC. Groups are as follows: Vehicle-PBS (white bars; $n=15$ ) and $1.0 \mu \mathrm{g} \mathrm{NE}$ (black bars; $n=16) A$, Mean latencies, in seconds ( \pm SEM), to enter the shock compartment during the retention test. $B$, Mean number of shocks ( \pm SEM) administered for animal to remain in the safe compartment for $200 \mathrm{sec}^{*} p<0.05$ compared with the vehicle controls.

IA are different forms of learning with different neurobiological substrates (Wilensky et al., 2000).

IA and CFC training are similar, because animals receive footshock in a particular context and are not allowed to make instrumental escape or avoidance responses during the training session. However, the IA training and testing procedures typically used differ from those used in CFC, because animals trained in IA are allowed to enter the place in the apparatus where the training shock is delivered or step down from a platform to a grid floor where shock is delivered. In retention tests, latencies to reenter the shock area or to step down to the grid floor are used to assess memory of the training. In both of the present experiments, CFC training procedures were used. Because the rats were placed in the compartment where shock was delivered and retained there during the CFC training, there was no opportunity for the learning of any escape or avoidance response. Also, in both experiments, the rat's latency to enter the shock compartment was used to assess retention of the training, and in both experiments, rats given post-training intra-BLA infusions of NE $(1.0 \mu \mathrm{g})$ had entrance latencies that were longer than those of the vehicle controls. Thus, the present findings on the basis of experiments using CFC trainotremorine enhance CFC retention as assessed by freezing, as well as by increased latencies to enter a compartment where shock had been received (Vazdarjanova and McGaugh, 1999). Histaminerenhance and impair retention, respectively, as assessed by freezing (Passani et al., 2001; Cangioli et al., 2002).

In view of the present findings, as well as those of other studies reporting that post-training infusions of drugs affecting several neurotransmitter and neuromodulatory systems enhance or impair the consolidation of memory for CFC, it is not clear why Wilensky et al. (2000) failed to find impairment of CFC with intra-BLA infusions of muscimol administered after CFC. It seems unlikely that muscimol effects in the BLA act through mechanisms unrelated to those of NE in view of the evidence that noradrenergic activation (Introini-Collison et al., 1994; Hatfield et al., 1999). The percent freezing by control animals in the Wilensky et al. (2000) study was substantially greater than that of the control animals in the present study (experiment 1). Such findings suggest that the susceptibility of CFC to post-training alterations in BLA functioning vary with the CFC training conditions. It might be that higher doses of muscimol are required to induce memory impairment under CFC conditions that result in substantial freezing. However, even under CFC conditions that produce substantial freezing, retention, as assessed by freezing, is impaired by temporarily inactivating the BLA after training with infusions of TTX or lidocaine (Sacchetti et al., 1999; Vazdarjanova and McGaugh, 1999). Comparable effects of TTX and lidocaine infusions are found in studies using IA (Bucherelli et al., 1992; Parent and McGaugh, 1994).

Overall, these findings indicate that neither the specific training methods nor the testing methods used in CFC and IA experiments are critical in enabling the modulation of memory consolidation by post-training treatments affecting BLA functioning. In particular, the response made before receiving the shock in IA training plays no critical role. Such findings are consistent with the evidence that post-training intra-BLA infusions of noradrenergic, cholinergic, or GABAergic agonists and antagonists modulate the consolidation of memory of other types of training that do not use footshock, including water-maze spatial learning, con-
intra-BLA infusions of the muscarinic cholinergic agonist oxgic agonists and antagonists administered into the BLA after CFC GABAergic influences on memory consolidation are mediated by 
ditioned taste aversion, conditioned place preference (CPP), and change in reward magnitude (Salinas and McGaugh, 1996; Hatfield and McGaugh, 1999; Schroeder and Packard, 2002) (M. I. Miranda, R.T. LaLumiere, T. V. Buen, F. Bermudez-Rattoni, and J. L. McGaugh, unpublished observations). The findings of the effects of post-training intra-BLA infusions on CPP (Schroeder and Packard, 2002) are of particular relevance because animals given CPP training are typically placed in an apparatus and allowed to learn that the context is associated with a reward. Thus, the Pavlovian training procedures are like those of CFC but differ in the reinforcement associated with the context (i.e., a reward rather than a footshock). Animals' preference for the context associated with the reward is typically used as an index of memory of the Pavlovian CPP training. Thus, the present findings are consistent with those of other experiments using CPP training as well as CFC training in indicating that post-training intra-BLA drug infusions influence the consolidation of memory for Pavlovian training experiences.

Because memory for training in these various kinds of tasks is known to involve BLA interactions with other brain regions, including the caudate nucleus, hippocampus, and entorhinal cortex, the present findings are also consistent with other evidence indicating that post-training alteration of BLA functioning modulates memory through influences of the BLA on consolidation occurring in other brain regions (Packard et al., 1994; McGaugh, 2002).

\section{References}

Brioni JD, Nagahara AH, McGaugh JL (1989) Involvement of the amygdala GABAergic system in the modulation of memory storage. Brain Res 487:105-112.

Bucherelli C, Tassoni G, Bures J (1992) Time-dependent disruption of passive avoidance acquisition by post-training intra-amygdala injection of tetrodotoxin in rats. Neurosci Lett 140:213-234.

Cangioli I, Baldi E, Mannaioni PF, Bucherelli C, Blandina P, Passani MB (2002) Activation of histaminergic $\mathrm{H}_{3}$ receptors in the rat basolateral amygdala improves expression of fear memory and enhances acetylcholine release. Eur J Neurosci 16:521-528.

Ferry B, McGaugh JL (1999) Clenbuterol administration into the basolateral amygdala post training enhances retention in an inhibitory avoidance task. Neurobiol Learn Mem 72:8-12.

Ferry B, Roozendaal B, McGaugh JL (1999a) Basolateral amygdala noradrenergic influences on memory storage are mediated by an interaction between $\beta$ - and $\alpha$-adrenoceptors. J Neurosci 19:5119-5123.

Ferry B, Roozendaal B, McGaugh JL (1999b) Role of norepinephrine in mediating stress hormone regulation of long-term memory storage: a critical involvement of the amygdala. Biol Psychiatry 46:1140-1152.

Hatfield T, McGaugh JL (1999) Norepinephrine infused into the basolateral amygdale post-training enhances retention in a spatial water maze task. Neurobiol Learn Mem 71:232-239.

Hatfield T, Spanis C, McGaugh JL (1999) Response of amygdalar norepinephrine to footshock and GABAergic drugs using in vivo microdialysis and HPLC. Brain Res 835:340-345.

Helmstetter FJ, Bellgowan PS (1994) Effects of muscimol applied to the basolateral amygdala on acquisition and expression of contextual fear conditioning in rats. Behav Neurosci 108:1005-1009.

Introini-Collison IB, Nagahara AH, McGaugh JL (1989) Memory enhance- ment with intra amygdala post-training naloxone is blocked by concurrent administration of propranolol. Brain Res 276:94-101.

Introini-Collison IB, Castellano C, McGaugh JL (1994) Interaction of GABAergic and beta noradrenergic drugs in the regulation of memory storage. Behav Neural Biol 61:150-155.

Izquierdo I, da Cunha C, Rosat R, Jerusalinsky D, Ferreira MB, Medina JH (1992) Neurotransmitter receptors involved in post-training memory processing by the amygdala, medial septum, and hippocampus of the rat. Behav Neural Biol 58:16-26.

Maren S, Aharonov G, Fanselow MS (1996) Retrograde abolition of conditional fear after excitotoxic lesions in the basolateral amygdala of rats: absence of a temporal gradient. Behav Neurosci 110:1365-1374.

McGaugh JL (2002) Memory consolidation and the amygdala: a systems perspective. Trends Neurosci 25:456.

McIntyre CK, Hatfield T, McGaugh JL (2002) Amygdala norepinephrine levels after training predict inhibitory avoidance retention performance in rats. Eur J Neurosci 16:1223-1226.

Muller J, Corodimas KP, Fridel Z, LeDoux JE (1997) Functional inactivation of the lateral and basal nuclei of the amygdala by muscimol infusion prevents fear conditioning to an explicit conditioned stimulus and to contextual stimuli. Behav Neurosci 111:683-691.

Packard MG, Cahill L, McGaugh JL (1994) Amygdala modulation of hippocampal-dependent and caudate nucleus-dependent memory processes. Proc Natl Acad Sci USA 91:8477-8481.

Parent MB, McGaugh JL (1994) Post-training infusion of lidocaine into the amygdala basolateral complex impairs retention of inhibitory avoidance training. Brain Res 661:97-103.

Passani MB, Cangioli I, Baldi E, Bucherelli C, Mannaioni PF, Blandina P (2001) Histamine $\mathrm{H}_{3}$ receptor-mediated impairment of contextual fear conditioning and in-vivo inhibition of cholinergic transmission in the rat basolateral amygdala. Eur J Neurosci 14:1522-1532.

Paxinos G, Watson C (1997) The rat brain in stereotaxic coordinates, Ed 3. San Diego: Academic.

Power AE, Thal LJ, McGaugh JL (2002) Lesions of the nucleus basalis magnocellularis induced by 192 IgG-saporin block memory enhancement with post-training norepinephrine in the basolateral amygdala. Proc Natl Acad Sci USA 19:2315-2319.

Quirarte GL, Roozendaal B, McGaugh JL (1997) Glucocorticoid enhancement of memory storage involves noradrenergic activation in the basolateral amygdala. Proc Natl Acad Sci USA 94:14048-14053.

Quirarte GL, Galvez R, Roozendaal B, McGaugh JL (1998) Norepinephrine release in the mygdala in response to footshock and opioid peptidergic drugs. Brain Res 808:134-140.

Sacchetti B, Lorenzini CA, Baldi E, Tassoni G, Bucherelli C (1999) Auditory thalamus, dorsal hippocampus, basolateral amygdala and perirhinal cortex role in the consolidation of conditioned freezing to context and to acoustic conditioned stimulus in the rat. J Neurosci 19:9570-9578.

Salinas JA, McGaugh JL (1996) The amygdala modulates memory for changes in reward magnitude: involvement of the amygdaloid GABAergic system. Behav Brain Res 80:87-98.

Schroeder JP, Packard MG (2002) Post-training intra-basolateral amygdala scopolamine impairs food- and amphetamine-induced conditioned place preferences. Behav Neurosci 116:922-927.

Vazdarjanova A, McGaugh JL (1998) Basolateral amygdala is not critical for cognitive memory of contextual fear conditioning. Proc Natl Acad Sci USA 95:15003-15007.

Vazdarjanova A, McGaugh JL (1999) Basolateral amygdala is involved in modulating consolidation of memory for classical fear conditioning. J Neurosci 19:6615-6622.

Wilensky AE, Schafe GE, LeDoux JE (2000) The amygdala modulates memory consolidation of fear-motivated inhibitory avoidance learning but not classical fear conditioning. J Neurosci 20:7059-7066. 